NASA-TM-112523

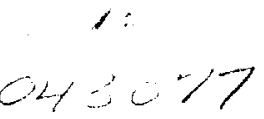

\title{
INTERNAL LUMINOSITY DISTRIBUTION OF BRIGHT GAMMA-RAY BURSTS AND ITS RELATION TO DURATION AND SPECTRAL HARDNESS
}

\author{
JOHN M. HORACK ${ }^{1}$ AND JON HAKKILA ${ }^{2}$ \\ Received 1996 July 1 ; accepted 1996 October 17
}

\begin{abstract}
We present first results from a comprehensive investigation into the distribution of luminosity within the 50 brightest cosmic gamma-ray bursts detected by the Burst and Transient Source Experiment (BATSE). The internal luminosity function $\psi(L)$ is defined such that the quantity $\psi(L) d L$ represents the fraction of total emission time during which the burst possesses a luminosity between $L$ and $L+d L$. For these brightest bursts, the $\psi(L)$ functions are quasi-power-law-like and decrease in amplitude with increasing luminosity. Through investigation of both individual $\psi(L)$ distributions and data from the ensemble of bursts, we demonstrate a high probability for correlation between the shape of the internal luminosity function as measured by the average logarithmic slope and the burst duration as measured by the $T_{90}$ parameter and, with lower significance, between the shape of $\psi(L)$ and the burst photon-fluence spectral index. We furthermore demonstrate a correlation between burst hardness ratio and duration in these brightest bursts which is opposite to that of the entire gamma-ray burst ensemble.

Subject headings: gamma rays: bursts
\end{abstract}

\section{INTRODUCTION}

The brightness distribution of cosmic gamma-ray bursts that is observed with the Burst and Transient Source Experiment (BATSE) (Fishman et al. 1989; Horack 1991) contains information pertaining to both the radial distribution of the burst sources and the distribution of bursts' peak luminosities. We have learned with BATSE that the distribution of bright bursts is consistent with a population of objects distributed homogeneously throughout Euclidean space, while at lower brightnesses the distribution deviates from homogeneity and there is a paucity of weak bursts. This behavior is attributed to an inhomogeneous distribution of burst sources or to cosmological effects (Meegan et al. 1992). Often these data are presented in integral form, with brightness $P$ along the ordinate and the total number of bursts exceeding a certain brightness $\mathscr{N}(>P)$ along the abscissa.

Mathematically, the integral brightness distribution can be represented for a Euclidean distribution of sources as

$$
\mathscr{N}(>P)=4 \pi \int_{0}^{\infty} \phi(L) \int_{0}^{(L / 4 \pi P)^{1 / 2}} n(r) r^{2} d r d L,
$$

where $n(r)$ is the volume number density of burst sources and $\phi(L)$ is the distribution of peak burst luminosities. The upper limit of integration on the $r$ integral reflects the fact that bursts having a given peak luminosity $L$ will be observed with peak brightness larger than $P$ only if their distance is less than the value $(L / 4 \pi P)^{1 / 2}$. From equation (1) it is easy to demonstrate that in the case where $n(r)$ is a constant, the integral brightness distribution will display a logarithmic slope of $-3 / 2$, regardless of the bursts' peak luminosity function $\phi(L)$.

The measure of brightness adopted for use in the integral brightness distribution is that of the peak burst flux, measured in units of photons $\mathrm{cm}^{-2} \mathrm{~s}^{-1}$ in the energy range $50-300 \mathrm{keV}$ over a predetermined time interval of either $0.064,0.256$, or $1.024 \mathrm{~s}$. By studying the distribution of peak

\footnotetext{
${ }^{1}$ Space Sciences Laboratory, NASA/MSFC, ES-84, Huntsville, AL 35812 .

${ }^{2}$ Department of Physics and Astronomy, Mankato State University, Mankato, MN 56002.
}

burst brightnesses, one can learn about the peak luminosities of the detected bursts.

Indeed, in recent years a number of papers have appeared in the literature dealing with the properties of the burst peak luminosity distribution $\phi(L)$, parameterized as a power law with a variety of spectral indices. Horack, Emslie, \& Meegan (1994) demonstrate that in the context of a Euclidean distribution of sources, $80 \%$ of the bursts detected by BATSE possess peak luminosities that span a range of 12 or less, regardless of the particular form of $n(r)$ or logarithmic slope of the $\phi(L)$ function. Ulmer \& Wijers (1995) utilize a different analysis technique to obtain a consistent result. By incorporating bursts detected by the Pioneer Venus Orbiter spacecraft, Ulmer, Wijers, \& Fenimore (1995) further restrict this range of luminosity, confining $90 \%$ of the detected bursts to a peak luminosity range of $\sim 5$ or less.

Hakkila et al. $(1995,1996)$ demonstrate that while the range of luminosity for the detected bursts is indeed narrow, there are three general classes of peak luminosity functions that can satisfy the constraints imposed by the BATSE brightness distribution. Two of these allow formally infinite ranges of peak luminosity. The first of these is the so-called $L_{\min }$-dominated case, where the peak luminosity function $\phi(L)$ is allowed to range from some finite lower limit $L_{\min }$ to infinity but must have a logarithmic slope steeper (less) than -2.5 . In this case, most of the detected bursts are located in a narrow range just slightly above the value $L_{\min }$. The second case is the $L_{\max }$-dominated case, where the peak luminosity function is constrained to be nonzero only below a maximum limiting value $L_{\max }$ and can only have a logarithmic slope that is shallower (greater) than -1.8 . In this scenario most of the detected bursts possess luminosities just slightly less than $L_{\max }$. The third case consists of peak luminosity distributions with logarithmic slope between -1.8 and -2.5 . Hakkila et al. $(1995,1996)$ demonstrate that these $\phi(L)$ must possess a finite $L_{\max } / L_{\min }$, again with a majority of the detected bursts confined to a narrow range of peak luminosities. Horack et al. (1996) demonstrate that these results can be obtained analytically and that the limiting logarithmic slopes found in Hakkila et al. (1995, 
1996) for the peak luminosity distribution are simply a consequence of the maximum and minimum logarithmic slopes displayed by the BATSE peak brightness distribution.

Each of these studies deals with the distribution of peak luminosities. Insofar as they explore the physics of the burster mechanism, they therefore relate only to the burst at one particular time in its evolution, the time at which the luminosity is a maximum. However, burst profiles are quite complex and vary significantly between bursts. It is possible that our understanding of the bursts may be furthered by studying the burst energy output throughout each of the burst events, rather than by examining the ensemble distribution obtained by selecting each detected burst at its peak energy production rate. We therefore present here the first results of our study into the internal luminosity distribution of gamma-ray bursts. To explicitly distinguish between the peak luminosity distribution and the internal luminosity distribution, we denote the latter function as $\psi(L)$, whereas the peak luminosity distribution is denoted as $\phi(L)$.

\section{DEFINING THE INTERNAL LUMINOSITY DISTRIBUTION} $\psi(L)$

The internal luminosity distribution is measured from the burst time profile. In principle, the quantity $\psi(L) d L$ represents the fraction of time during which the burst possesses a luminosity between $L$ and $L+d L$. In practice, since the distance to any burst remains unknown, the scaling of the luminosity will not be absolute. We adopt the BATSE $0.064 \mathrm{~s}$ discriminator science (DISCSC) count-rate data (e.g., Horack 1991) as the data of choice for computing the internal luminosity distribution, and we confine ourselves to the energy range of $50-300 \mathrm{keV}$. Thus our raw data used to compute the distribution possess the instrumental units of counts $\mathrm{s}^{-1}$, rather than the physical units of photons $\mathrm{s}^{-1}$. To utilize physical units requires the adoption of a photon spectral model, detector response matrix, and spectral deconvolution of each $64 \mathrm{~ms}$ interval of the burst under examination. For our purposes, it is sufficient to assume an approximately diagonal response matrix for the intensities and energies used here and utilize the more convenient count-space data in computing $\psi(L)$.

To obtain the $\psi(L)$ distribution for a given burst, a quadratic background fit is subtracted from the detected time profile. The resulting burst light curve is then normalized to the maximum intensity level exhibited during the event. From this normalized, background-subtracted profile, a frequency-brightness distribution is obtained from the $0.064 \mathrm{~s}$ intensity intervals, indicating the number of times a given intensity level was observed in the $64 \mathrm{~ms}$ time intervals. Time intervals when the background-subtracted intensity is either less than $3 \sigma$ above background or less than 0.01 times the maximum intensity are not included to avoid the introduction of noise into the distribution. This frequency distribution is then recast in the form of a distribution function and normalized to the total amount of time the burst is observed to have intensity above the threshold criterion stated previously. In discretized form, therefore, the normalization for $\psi(L)$ can be written as

$$
\sum_{i} \psi(L)_{i} \Delta L_{i}=1
$$

and the resulting $\psi(L)$ distribution represents the fraction of the total detection time during which the burst was observed to have a luminosity between $L$ and $L+d L$, with the luminosity normalized to the maximum of the event.

Before investigating the internal luminosity distributions observed by BATSE, it is illustrative to consider the functions that can be obtained from simple, analytic models to burst pulse profiles. General features and behaviors found in the internal luminosity distributions of simple profiles can then be better understood in the context of the more complicated physical bursts. We employ the variable $I$ as the measure of luminosity when dealing with these synthetic pulse shapes to avoid confusion between these and actual gamma-ray burst profiles.

\subsection{Gaussian Profile}

To begin, we shall examine the internal luminosity distribution function obtained from a Gaussian profile. This particular profile is modeled as

$$
I(t)=I_{0} \exp \left[\frac{-\left(t-t_{0}\right)^{2}}{2 \sigma^{2}}\right] .
$$

Our interest in this shape is for the purposes of illustration, as its ability to represent the types of time profiles actually observed from gamma-ray bursts is quite limited.

We identify $t_{1}$ and $t_{4}\left(t_{1}<t_{4}\right)$ as the times when the intensity of the profile is equal to 0.01 and $t_{2}$ and $t_{3}\left(t_{2}<t_{3}\right)$ as the times when the intensity is equal to $I_{1}$. These times are shown superposed on the profile (3) in Figure 1 where we have adopted values of $I_{0}=1.0, t_{0}=50$ units, and $\sigma=15$ units.

According to the definition of the internal luminosity distribution,

$$
\int_{0.01}^{I_{1}} \psi(I) d I=\frac{t_{2}-t_{1}+t_{4}-t_{3}}{t_{4}-t_{1}} .
$$

Equation (4) simply indicates the amount of time the profile is observed to have intensity between 0.01 and $I_{1}$, normalized to the total time observed above the level 0.01 . Since in this case the time profile is symmetric,

$$
t_{2}-t_{1}=t_{4}-t_{3}
$$

allowing us to rewrite equation (4) as

$$
\int_{0.01}^{I_{1}} \psi(I) d I=\frac{2\left(t_{2}-t_{1}\right)}{t_{4}-t_{1}} .
$$

To proceed further, we first solve equation (3) for $t$, writing it as a function of $I$, yielding

$$
t=\left[-2 \sigma^{2} \ln \left(\frac{I}{I_{0}}\right)\right]^{0.5}+t_{0} .
$$

We may now use equation (7) in equation (6) to obtain an expression involving $\psi(I)$ on the left-hand side and the intensity $I$ on the right-hand side. Specifically, we obtain

$$
\begin{aligned}
\int_{0.01}^{I_{1}} \psi(I) d I= & \frac{2}{t_{4}-t_{1}} \\
& \times\left\{\left[-2 \sigma^{2} \ln \left(\frac{I_{1}}{I_{0}}\right)\right]^{0.5}\right. \\
& \left.-\left[-2 \sigma^{2} \ln \left(\frac{0.01}{I_{0}}\right)\right]^{0.5}\right\} .
\end{aligned}
$$




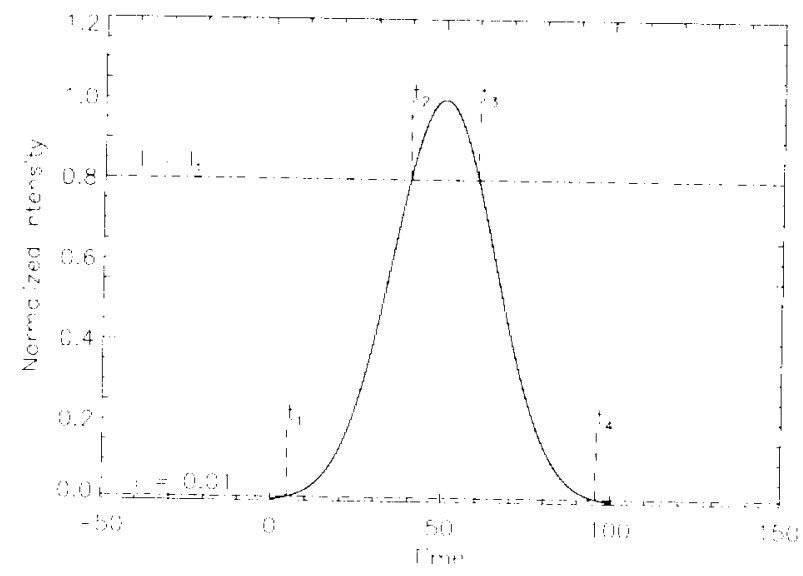

FIG. 1.-Sample Gaussian pulse profile obtained from eq. (3). Times $t_{1}$ and $t_{4}$ are shown where the intensity equals 0.01 of the maximum. Similarly, $t_{2}$ and $t_{3}$ indicate the times where the intensity is equal to $I_{1}$.

Finally by differentiation of the preceding equation we obtain the final result :

$$
\psi(I)=\frac{1}{t_{4}-t_{1}} \frac{\left[-2 \sigma^{2} \ln \left(I / I_{0}\right)\right]^{-0.5}}{I} .
$$

The measured internal luminosity distribution for the profile in Figure 1 is shown in Figure 2 as a histogram. The $\psi(I)$ distribution here (and in all subsequent figures) is presented in logarithmic space both for clarity and simplicity, although the discussion to this point has presented the $\psi(I)$ distribution per unit luminosity. The error bars are propagated from the frequency distribution, where we assume Poisson counting statistics and compute the error bar simply as the square root of the number of times each intensity interval was sampled from the Gaussian form. Below the histogram, we have plotted equation (9) as a dot-dashed curve with the normalization modified to place the curve below the histogram for clarity. Both the analytical and measured $\psi(I)$ distributions are characterized by a quasipower law for low values of $I$ and an upturn in the distribution at large values of $I$ due to the flat-topped nature of the Gaussian profile. That the logarithmic slope of the $\psi(I)$ dis-

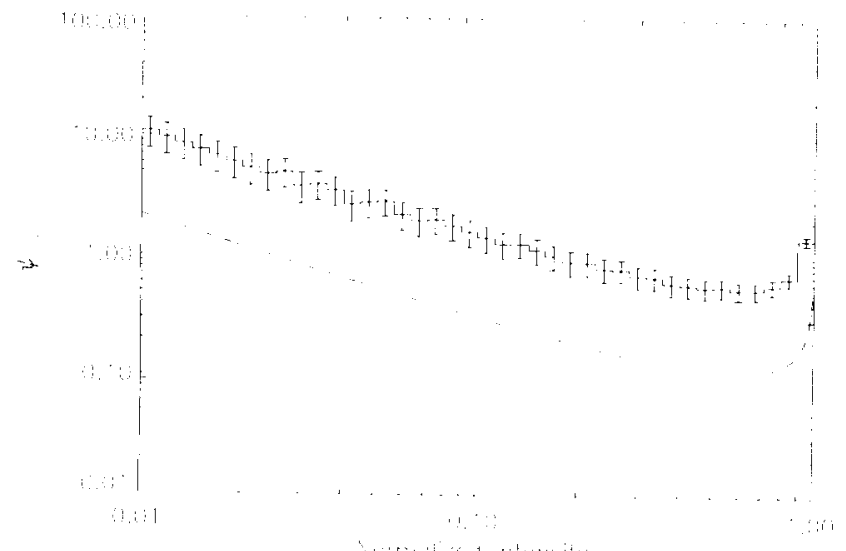

FIG. 2.-Measured internal luminosity distribution $\psi(I)$ from the synthetic Gaussian pulse profile of Fig. 1 (histogram). Dot-dashed curve, plot of eq. (9), the analytically derived $\psi(I)$ for this profile shape. The normalization has been offset for clarity. tribution is one measure of the "peakedness" of a given profile shape is a feature that will be useful in further discussions.

\subsection{An Asymmetric Profile}

The preceding profile is a gross oversimplification of what one actually observes in gamma-ray bursts. Bursts, as well as the pulses within bursts, display a tremendous range of morphologies. Some pulses within bursts can be significantly spiked, while others may display a more rounded behavior (see Fishman \& Meegan 1995 for a variety of examples). It is also well known that both bursts and the pulses within bursts are asymmetric (Link, Epstein, \& Priedhorsky 1993; Nemiroff et al. 1994) in time, with the characteristic rise time less than the decay time of a pulse. Having successfully demonstrated the simplest case $\psi(I)$ previously, it is illustrative to further the analysis of the preceding section utilizing a more complex profile parameterization that provides for a variety in the "spikedness" of the pulses, as well as an overall asymmetry similar to that observed in nature.

We borrow the parameterization of Norris et al. (1993a, 1993b) and Davis et al. (1993):

$$
I(t)=I_{0} \exp \left[-\left(\frac{\left|t-t_{0}\right|}{\sigma_{r, f}}\right)^{v}\right] .
$$

In this expression, $t_{0}$ is the time of maximum intensity $I_{0}$, $\sigma_{r, f}$ is the characteristic width of the pulse rise $(r)$ and pulse decay $(f)$, and $v$ is the "peakedness" parameter. Low values of $v$ generate pulses that are more strongly spiked, and larger values of $v$ result in shapes that are more rounded or level near the maximum.

Figure 3 contains an example of a profile generated using equation (10), with parameters $I_{0}=1, t_{0}=50, \sigma_{r}=0.5$, $\sigma_{f}=6.0$, and $v=1.0$. It is apparent that equation (4) is valid for this particular profile as well as the previous case; however, here we cannot make the same simplifying assumption based on symmetry. It is, however, straightforward to proceed as before in determining the functional form for $\psi(I)$ by solving equation (10) for $t$ explicitly and substituting these results into equation (4). This analysis

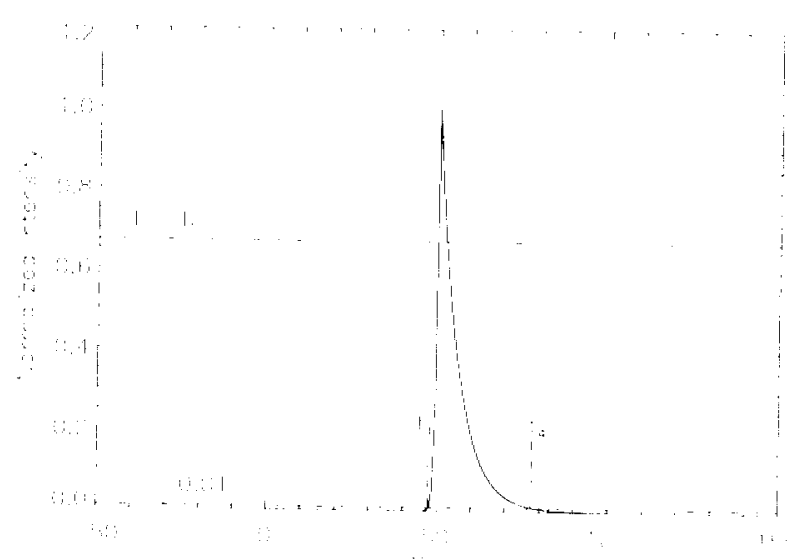

FIG. 3.-Sample asymmetric profile obtained from eq. (10), with parameters $\sigma_{r}=0.5, \sigma_{f}=6.0, v=1$, and $I_{0}=1.0$. This more general form allows for variations in the characteristic rise and decay times, as well as the "spikedness" of the profile. 


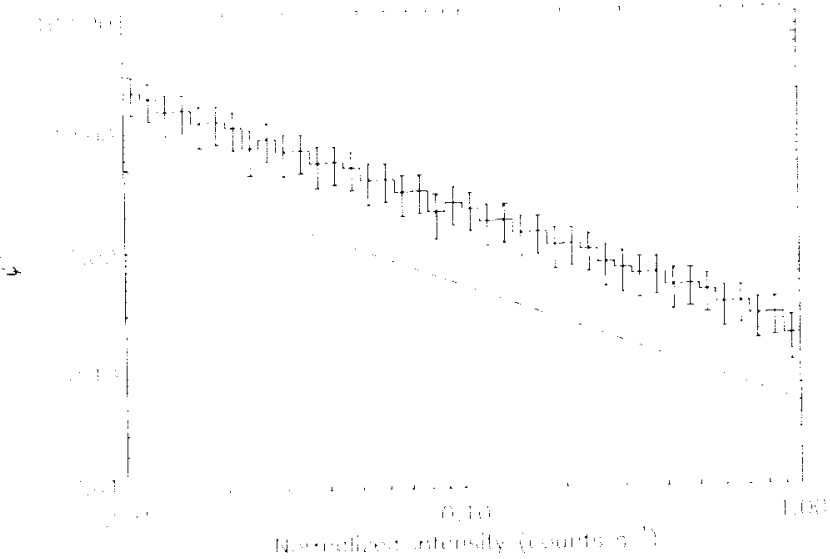

Fig. 4--Measured internal luminosity distribution for the profile in Fig. 3 (histogram). Dot-dashed curve, plot of eq. (11), the derived analytical form of the internal luminosity distribution for this parameterized profile shape. The normalization is intentionally offset for clarity in the figure.

results in the explicit expression for $\psi(I)$ :

$$
\psi(I)=\frac{\sigma_{r}+\sigma_{f}}{t_{4}-t_{1}} \frac{1}{I v}\left[-\ln \left(\frac{I}{I_{0}}\right)\right]^{(1 / v)-1} .
$$

In Figure 4, we present the $\psi(I)$ distribution measured for the profile in Figure 3 as a histogram and plot equation (11) as a dot-dashed line. We again have modified the normalization in equation (11) to separate the curve from the histogram to provide better clarity in the figure.

This distribution is markedly different from the previous case; most noticeable is the absence of the upturn in $\psi(I)$ for large $I$ that was present in the Gaussian profile. Figure 4 displays a distribution that behaves as a power law of index -1 for all intensities shown. Inspection of equation (11) shows that this is a direct result of the value of $v=1$ chosen for this particular example. With $v=1$, the exponent is zero, leaving a simple $1 / I$ dependence.

In the general case of this parameterization, the logarithmic slope of the distribution can be written as

$$
\frac{d \ln \psi}{d \ln I}=\frac{I}{\psi} \frac{d \psi}{d I}=-1\left[1+\frac{(1 / v)-1}{-\ln \left(I / I_{0}\right)}\right] .
$$

For $v=1$, we again observe the logarithmic slope equal to -1 for all 1 . Thus the example above is a special case. For very large values of $v$, the distribution behaves quite differently. In the limit of large $v$, one has behavior like

$$
\psi(I) \sim-\left[1+\frac{1}{\ln \left(I / I_{0}\right)}\right] .
$$

This provides a logarithmic slope of approximately -0.8 at low $I$ and can eventually become positive with increasing $I$, allowing the distribution to display an upturn for sufficiently large $v$ and $I$. As large values of $v$ produce profiles with more flat-topped peaks, one expects that the $\psi(I)$ distribution should display an upturn in the regions of high intensity, as the profile is observed to spend a proportionally larger amount of time in this region. Conversely, when $v$ is very small, one has a very sharp peak. We observe from equation (12) that small values of $v$ can result in slopes that are arbitrarily small (steep) with increasing $I$. Thus in addition to a possible upturn in the distribution, one can also have a downturn depending on the value of $v$. Extremely sharp time profiles spend proportionally less time near the peak, so one expects that in these cases the $\psi(I)$ distribution should show diminished activity at large intensities as the equation predicts. We therefore identify three regimes for this parameterization, $v>1, v=1$, and $v<1$, each of which results in different behavior at the high-intensity end of the $\psi(I)$ distribution.

\subsection{The "Real" World}

Although more complex than the Gaussian case of $\S 2.1$, the preceding asymmetric time profile with a variable peakedness parameter is still quite distant from an accurate representation of the overall burst profiles observed by BATSE. Bursts consist of many varied pulses, some of which can overlap and display different behavior in different energy regions. The resulting $\psi(I)$ distribution will be a conglomeration of each individual pulse distribution added together in an incoherent manner. Unfortunately, the combination of just two separate pulses into a single time profile complicates the analytic approach to $\psi(I)$ enormously, resulting in nontrivial calculations of the $\psi(I)$ profile shape. In many simple cases, an analytic solution cannot be derived.

However, it is illustrative to consider one additional simple example before proceeding to look at actual burst profiles. Figure $5 a$ presents a simple time profile consisting

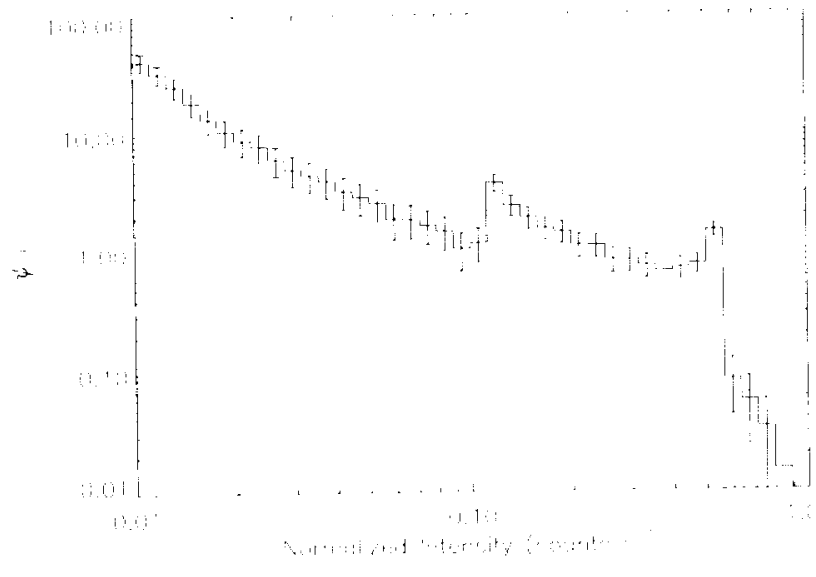

FIG. $5 b$

FIG. $5 a$

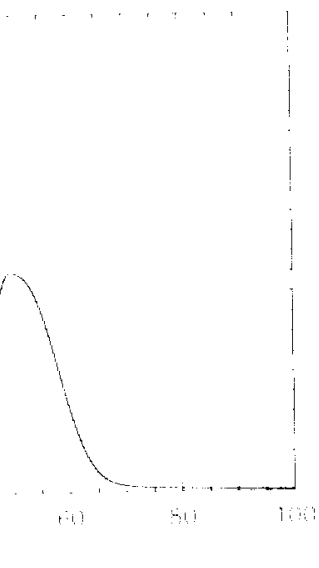
FIG. 5.-(a) More complex time profile involving both a peaked and rounded pulse. (b) The internal luminosity distribution measured for this profile. Although more complex than the previous cases, salient features and characteristics of each individual pulse are still visible in the overall internal luminosity distribution. 

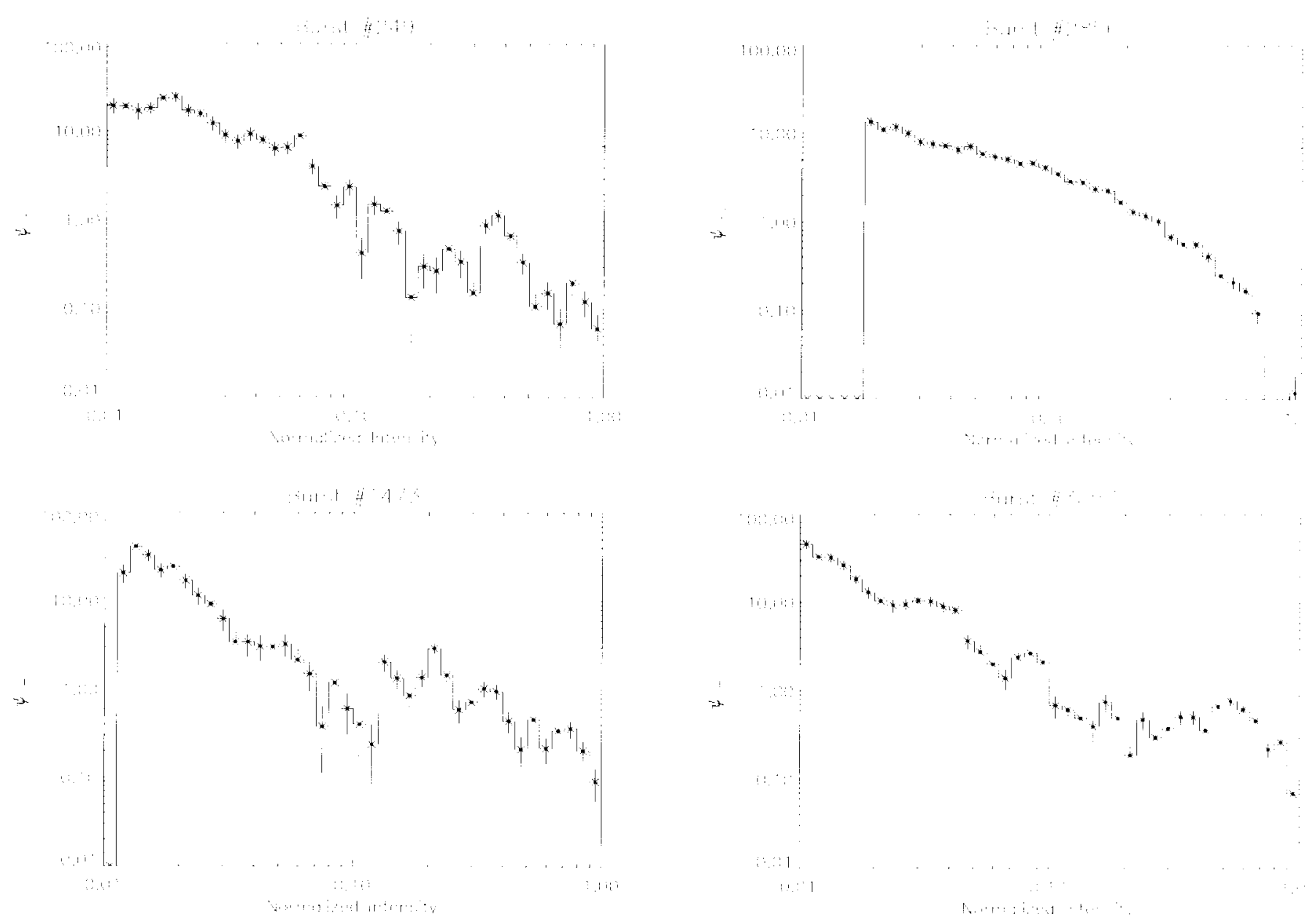

FIG. 6.-Internal luminosity $\psi(L)$ distributions for four of the 50 brightest gamma-ray bursts detected by BATSE. Intensity, normalized to the peak of the burst, is plotted along the $x$-axis. The quantity $\psi(L) d L$ represents the fraction of the total detection time the burst was observed to have intensity (luminosity) between $L$ and $L+d L$.

of two overlapping pulses, the second with half the amplitude of the first. Each individual pulse was generated using the functional form of equation (10), the first with parameters $t_{0}=30, \sigma_{r}=1, \sigma_{f}=2.25$, and $v=0.5$. For the second pulse, values of $t_{0}=50, \sigma_{r}=5, \sigma_{f}=10$, and $v=2.5$ were chosen. Thus the profile contains both a "spiked" and a "flat-topped" pulse profile. The $\psi(I)$ distribution measured for these pulses is shown in Figure $5 b$.

As expected, the $\psi(I)$ distribution is significantly more complex than either of the distributions obtained from individual pulses alone; however, salient features are still identifiable. First, one can still observe the contribution of the more intense pulse at the high-intensity end of the distribution. With the brighter peak possessing a small $(<1)$ value of $v$, the anticipated steep logarithmic slope can be observed for intensities above $I \sim 0.45$. At low intensities, the distribution is smooth and not quite power-law-like, receiving contributions in this area from the low-level intensities of both peaks. Just above a value of $I=0.1$, there is an abrupt increase in the value of $\psi(I)$. This is due to the presence of the interpulse region, where the burst is observed to spend a larger proportion of time compared to intensities just below this value. As $I$ increases, we observe the logarithmic slope of the $\psi(I)$ distribution increasing as well, eventually becoming positive between values of $I \approx 0.35-0.40$. This occurs because of the contribution of the more rounded (large v) secondary pulse and is similar to the behavior in Figure 2. The $\psi(I)$ distribution then displays an abrupt decrease in value, as one is now exploring intensities above the maximum of the secondary pulse. One can therefore observe both abrupt increases and decreases in the $\psi(I)$ distribution based on the structure of the time profile. The behavior in the remaining region of large $I$ is dominated by the spiked nature of the primary pulse, as noted previously.

\section{ANALYSIS OF THE 50 BRIGHTEST GAMMA-RAY BURSTS}

Having examined the internal luminosity distributions and their characteristic features found for a variety of synthetic time profile shapes, we now turn to the measurement of the internal luminosity distribution for actual gamma-ray bursts. For this analysis, we have examined the 50 brightest bursts as measured by the $1.024 \mathrm{~s}$ peak flux in the BATSE 3B catalog (Meegan et al. 1996). Of these 50 brightest bursts in the 3B catalog, 46 were usable for computation of $\psi(L)$. For one of the bursts, BATSE trigger No. 2151, the duration of the event was insufficient to obtain a reasonable number of data points in computing $\psi(L)$. Two others, No. 543 and No. 999, suffered from data problems, and an additional burst, No. 142, occurred during a magnetospheric electron precipitation event (e.g., Horack et al. 1991), so that the background was not sufficiently well behaved to perform a reliable analysis. 
TABLE 1

Summary Data Obtained from the $\psi(L)$ Distributions for the 50 Brightest BatSE Gamma-RaY BurSTS

\begin{tabular}{|c|c|c|c|c|c|c|c|c|c|}
\hline Trigger & $\alpha$ & $\chi_{v}^{2}$ & $v$ & $\langle L\rangle$ & $\delta(\langle L\rangle)$ & HR & $\delta(\mathrm{HR})$ & $T_{90}$ & $\delta\left(T_{90}\right)$ \\
\hline 2083 & 1.175 & 2.45 & 38 & 0.166 & 0.012 & 3.38 & 0.01 & 15.2 & 0.2 \\
\hline 1609 & 1.200 & 4.16 & 18 & 0.156 & 0.018 & 3.09 & 0.02 & 12.7 & 3.2 \\
\hline 143 & 1.225 & 2.16 & 38 & 0.164 & 0.012 & 6.01 & 0.04 & 50.8 & 0.2 \\
\hline 2831 & 1.575 & 11.03 & 38 & 0.083 & 0.003 & 3.83 & 0.01 & 150.0 & 0.0 \\
\hline 1541 & 1.200 & 4.76 & 38 & 0.152 & 0.008 & 3.98 & 0.02 & 26.2 & 0.2 \\
\hline 249 & 1.400 & 6.70 & 38 & 0.141 & 0.007 & 3.47 & 0.01 & 28.5 & 0.9 \\
\hline 1085 & 1.075 & 7.32 & 18 & 0.239 & 0.013 & 2.20 & 0.01 & 19.2 & 0.2 \\
\hline 3057 & 1.400 & 10.27 & 38 & 0.199 & 0.007 & 5.26 & 0.01 & 34.9 & 0.5 \\
\hline 2329 & 1.150 & 6.60 & 38 & 0.166 & 0.008 & 4.83 & 0.02 & 22.1 & 0.1 \\
\hline 1625 & 1.075 & 3.06 & 38 & 0.188 & 0.011 & 4.65 & 0.02 & 16.1 & 0.1 \\
\hline 1473 & 1.025 & 9.85 & 36 & 0.205 & 0.010 & 4.06 & 0.02 & & $\ldots$ \\
\hline 2798 & 1.675 & 7.72 & 34 & 0.147 & 0.006 & 4.99 & 0.03 & 37.6 & 0.1 \\
\hline 2431 & 1.500 & 1.52 & 18 & 0.135 & 0.023 & 2.83 & 0.04 & 2.78 & 0.13 \\
\hline 2611 & 1.875 & 1.89 & 18 & 0.126 & 0.023 & 4.70 & 0.08 & 12.2 & 0.1 \\
\hline 2537 & 0.500 & 7.92 & 18 & 0.289 & 0.024 & 2.28 & 0.02 & 4.80 & 0.09 \\
\hline 2067 & 1.275 & 11.72 & 8 & 0.231 & 0.011 & 4.58 & 0.02 & 30.8 & 0.5 \\
\hline 1663 & 1.225 & 12.23 & 33 & 0.256 & 0.010 & 5.43 & 0.03 & 36.0 & 1.0 \\
\hline 3067 & 1.925 & 5.12 & 34 & 0.126 & 0.008 & 3.50 & 0.03 & 67.0 & 1.0 \\
\hline 1886 & 1.325 & 4.82 & 33 & 0.291 & 0.019 & 6.33 & 0.05 & 276.0 & 2.0 \\
\hline 1711 & 0.875 & 1.84 & 32 & 0.269 & 0.027 & 5.24 & 0.06 & 5.95 & 0.14 \\
\hline 451 & 1.250 & 2.31 & 15 & 0.263 & 0.017 & 1.67 & 0.02 & 15.2 & 2.4 \\
\hline 2856 & 1.625 & 40.28 & 26 & 0.143 & 0.003 & 7.34 & 0.03 & 120.0 & 0.0 \\
\hline 3138 & 1.450 & 5.62 & 32 & 0.216 & 0.022 & 2.78 & 0.04 & 5.18 & 0.09 \\
\hline 2953 & 1.225 & 1.67 & 14 & 0.210 & 0.014 & 3.40 & 0.03 & 16.8 & 0.8 \\
\hline 219 & 1.500 & 3.35 & 33 & 0.122 & 0.007 & 2.50 & 0.03 & 29.7 & 1.4 \\
\hline 3128 & 1.075 & 3.27 & 28 & 0.278 & 0.009 & 5.08 & 0.03 & 32.2 & 0.1 \\
\hline 2156 & 1.575 & 22.34 & 32 & 0.148 & 0.004 & 4.29 & 0.02 & 154.0 & 0.0 \\
\hline 1025 & 0.875 & 0.79 & 14 & 0.265 & 0.036 & 2.10 & 0.05 & 2.62 & 0.14 \\
\hline 2994 & 1.725 & 2.56 & 30 & 0.117 & 0.006 & 6.25 & 0.09 & 48.6 & 3.9 \\
\hline 1121 & 1.350 & 3.45 & 28 & 0.220 & 0.010 & 2.75 & 0.03 & & \\
\hline 1440 & 1.300 & 1.44 & 30 & 0.200 & 0.017 & 4.17 & 0.07 & 24.4 & 1.0 \\
\hline 1709 & 0.625 & 2.82 & 13 & 0.367 & 0.033 & 2.62 & 0.03 & 3.46 & 0.23 \\
\hline 105 & 1.325 & 3.44 & 14 & 0.210 & 0.022 & 1.55 & 0.14 & 5.18 & 0.18 \\
\hline 2090 & 1.200 & 2.06 & 13 & 0.237 & 0.015 & 3.24 & 0.04 & 38.1 & 0.1 \\
\hline 1157 & 1.600 & 1.34 & 29 & 0.179 & 0.013 & 3.45 & 0.04 & 171.0 & 2.0 \\
\hline 3115 & 1.500 & 3.37 & 27 & 0.213 & 0.013 & 5.16 & 0.09 & 45.3 & 0.1 \\
\hline 1141 & 0.725 & 4.47 & 28 & 0.335 & 0.014 & 2.46 & 0.02 & 20.1 & 0.5 \\
\hline 1122 & 1.400 & 4.34 & 31 & 0.183 & 0.009 & 3.19 & 0.03 & 18.8 & 1.0 \\
\hline 2855 & 1.475 & 13.25 & 27 & 0.247 & 0.008 & 6.51 & 0.05 & 42.5 & 1.8 \\
\hline 1484 & 2.450 & 2.42 & 14 & 0.118 & 0.016 & 2.77 & 0.06 & $\ldots$ & \\
\hline 2533 & 1.200 & 23.26 & 27 & 0.269 & 0.005 & 4.83 & 0.03 & 74.3 & 5.3 \\
\hline 2894 & 0.800 & 2.71 & 12 & 0.328 & 0.032 & 3.20 & 0.07 & 3.78 & 0.41 \\
\hline 2797 & 1.075 & 4.31 & 26 & 0.292 & 0.021 & 3.65 & 0.06 & 8.83 & 0.20 \\
\hline 1664 & 0.725 & 2.23 & 12 & 0.351 & 0.034 & 1.72 & 0.04 & 3.52 & 1.3 \\
\hline 2891 & 1.650 & 2.03 & 26 & 0.175 & 0.008 & 5.37 & 0.07 & 33.5 & 0.5 \\
\hline 1425 & 1.100 & 1.67 & 27 & 0.233 & 0.017 & 1.62 & 0.03 & 10.4 & 0.7 \\
\hline
\end{tabular}

Examples of four $\psi(L)$ distributions are shown in Figure 6. The error bars are propagated from the frequency distribution, where we assume Poisson counting statistics and compute the error bar simply as the square root of the number of times each intensity interval was sampled. The general features of the $\psi(L)$ functions shown in Figure 6 are found in all the bursts we have examined. Specifically, each of the $\psi(L)$ functions are quasi-power-law-like and decrease in value with increased intensity, demonstrating that the bursts spend proportionally more time at low luminosity levels than at high luminosity levels.

A variety of statistical measurements are made on each of the $\psi(L)$ functions obtained. Among these, a best-fit powerlaw exponent $\alpha$ is determined, based on minimization of the reduced $\chi^{2}$ statistic, in order to obtain a general description of the overall shape of the $\psi(L)$ function. We also compute the integral moments of the $\psi(L)$ distribution. The moments both offer a more complete description of the shape of the distribution and are useful in comparing the obtained $\psi(L)$ function with distributions of peak luminosities $\phi(L)$ that are compatible with the observed brightness distribution of bursts. Following the computation and analyses of $\psi(L)$ for the bursts individually, we then search the resulting data set for correlations among the different data values in an attempt to learn more about the behavior of the bursts.

Table 1 summarizes some of these computed data. Each of the 46 usable BATSE bursts are listed, ranked by decreasing intensity. The best-fit power-law index $\alpha$ to the $\psi(L)$ distribution is shown, along with the reduced $\chi^{2}$ and number of degrees of freedom in the fit. The average $\langle L\rangle$ of the $\psi(L)$ distribution, as well as the error in the mean, is also displayed in the table. Last, we also have incorporated data from the BATSE 3B catalog (Meegan et al. 1996), listing both the hardness ratio (HR) and its uncertainty, as well as the computed value of $T_{90}$ (e.g., Koshut et al. 1996) and its uncertainty, which is a measure of the burst duration. Several of the bursts listed in Table 1 do not have corresponding $T_{90}$ values in the BATSE $3 \mathrm{~B}$ catalog; thus no data appear for these bursts. 


\section{CORRELATIONS IN THE DATA}

The data in Table 1 show that a significant majority of the $\psi(L)$ functions found for the gamma-ray bursts are poorly represented by a powerlaw in the $\chi^{2}$ sense. However, despite these large values for the reduced $\chi^{2}$ statistic, the best-fit power-law index $\alpha$ can be used as a representative description of the average logarithmic slope of the internal luminosity function over the range of brightness studied, similar to the use of a hardness ratio to describe the general behavior of a burst's photon spectrum.

To search for correlations in the data, we employ the Spearman rank-order correlation test (e.g., Press et al. 1989). When applied, the nonparametric Spearman test returns a correlation value $r_{s}$ and the probability $P\left(r_{s}, N\right)$ that $N$ pairs of uncorrelated variables would yield a value of $r_{s}$ equally or more discrepant than the one obtained from the data set. A weakness of the method is that it does not provide information on the functional form of any possible correlation between variables, only the probability that a correlation of some kind is present. It also offers no indication for the origin of the correlation, whether due to an actual physical relationship between the variables or some systematic effect in the analysis.

A summary of the parameters that we have investigated and their corresponding $r_{s}$ and $P\left(r_{s}, N\right)$ values are shown in Table 2. A cursory inspection of the table shows several very small values of $P\left(r_{s}, N\right)$, indicating a high probability for the presence of a correlation. We believe that two of these, in particular, the correlations between the average luminosity $\langle L\rangle$ and the burst intensity, and between $\langle L\rangle$ and the best-fit exponent $\alpha$, are systematic.

For the former, as burst intensity decreases, the likelihood increases that 0.01 times the maximum intensity will be less than $3 \sigma$ above the fitted background. In this case, the lower limit on the measured $\psi(L)$ will not be 0.01 but some larger number. For a given shape of $\psi(L)$, as the lower limit of the measured distribution increases, the average must also increase as the upper limit to the distribution is fixed at a value of 1.0 .

The latter correlation has a higher significance. The Spearman $r_{s}$ value for these data is -0.751 , corresponding to a probability $P\left(r_{s}, N\right)$ of $1.79 \times 10^{-9}$, a strong indication of correlation between the data sets. If we consider a normalized power-law functional form over a fixed intensity range, an increase in the power-law exponent results in a

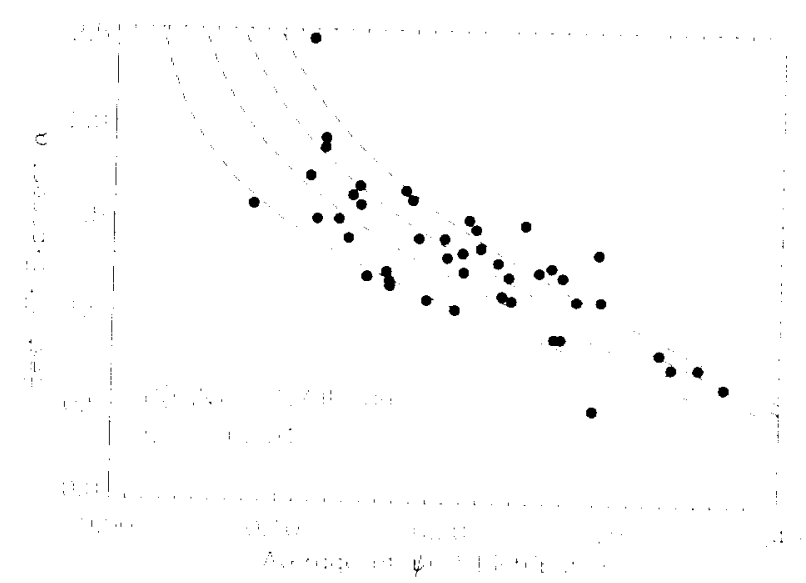

FiG. 7.-Filled circles, scatter plot of the average of the best-fit exponent $\alpha$ vs. the average $\langle L\rangle$ of the $\psi(L)$ distribution. Dashed lines, computed relationships from normalized power-law forms with varying $L_{\min }$ and a fixed $L_{\max }=1.0$. Low Spearman rank-order probability $P\left(r_{s}, N\right)$ indicates a very high probability for a correlation between these two variables, as
expected.

steepening of the shape of $\psi(L)$, which produces a lower value for the average $\langle L\rangle$.

In Figure 7, we present a scatter plot of the best-fit exponent $\alpha$ versus $\langle L\rangle$. The data are plotted as filled circles. The dashed lines overplotted on the figure are curves of $\alpha$ versus $\langle L\rangle$ calculated for power-law functional forms with different values of $L_{\mathrm{min}}$, ranging from 0.01 (lower left curve) to 0.04 (upper right curve) in steps of 0.01 , and a fixed $L_{\max }=$ 1.0. We observe that the data points are closely located along the theoretical curves. That the measured $\psi(L)$ distribution functions are not exact power laws has an effect on the location of the points relative to these curves, producing some scatter. However, the general agreement between the data and the curves offers validation of the first-order power-law description of the $\psi(L)$ functions used in this analysis.

\subsection{Best-Fit Exponent $\alpha$ versus $T_{90}$}

Having obtained the values of $T_{90}$ for these bursts from the BATSE 3B catalog (Meegan et al. 1996), we have plotted these values against the best-fit power-law index $\alpha$. These data are shown in Figure 8 . Those bursts in Table 1 that do not have $T_{90}$ values are omitted from this portion of the analysis. Application of the Spearman test to the data in

TABLE 2

Valuts of $r_{s}$ and $P\left(r_{s}, N\right)$ Obtained from Application of the SpEarman Rank-Order Test

\begin{tabular}{|c|c|c|c|c|c|}
\hline \multirow[b]{2}{*}{ VARIABLE } & \multicolumn{5}{|c|}{$\begin{array}{c}P\left(r_{s}, N\right) \\
r_{s} \\
\end{array}$} \\
\hline & Intensity & $T_{90}$ & Best-Fit Exponent & 3B Hardness Ratio & $\langle L\rangle$ \\
\hline Intensity & & $\begin{array}{c}6.22 \times 10^{-1} \\
0.0773\end{array}$ & $\begin{array}{c}9.12 \times 10^{-1} \\
0.1664\end{array}$ & $\begin{array}{c}2.16 \times 10^{-1} \\
0.1861\end{array}$ & $\begin{array}{c}3.38 \times 10^{-3} \\
-0.4832\end{array}$ \\
\hline$T_{90}$ & $\begin{array}{c}6.22 \times 10^{-1} \\
0.0773\end{array}$ & & $\begin{array}{c}4.71 \times 10^{-5} \\
0.5792\end{array}$ & $\begin{array}{c}1.32 \times 10^{-6} \\
0.6622\end{array}$ & $\begin{array}{c}-0.4832 \\
1.60 \times 10^{-2} \\
-0.3652\end{array}$ \\
\hline Best-Fit Exponent & $\begin{array}{c}9.12 \times 10^{-1} \\
0.1664\end{array}$ & $\begin{array}{c}4.71 \times 10^{-5} \\
0.5792\end{array}$ & & $6.58 \times 10^{-3}$ & $\begin{array}{c}1.79 \times 10^{-9} \\
-0.7513\end{array}$ \\
\hline 3B Hardness Ratio & $\begin{array}{c}2.16 \times 10^{-1} \\
0.1861\end{array}$ & $\begin{array}{c}1.32 \times 10^{-6} \\
0.6622\end{array}$ & $\begin{array}{c}6.58 \times 10^{-3} \\
0.4080\end{array}$ & 0.4080 & $\begin{array}{c}-0.7513 \\
1.56 \times 10^{-1} \\
-0.2127\end{array}$ \\
\hline$\langle L\rangle$ & $\begin{array}{c}3.38 \times 10^{-3} \\
-0.4832 \\
\end{array}$ & $\begin{array}{c}1.60 \times 10^{-2} \\
-0.3652\end{array}$ & $\begin{array}{c}1.79 \times 10^{-9} \\
-0.7513\end{array}$ & $\begin{array}{c}1.56 \times 10^{-1} \\
-0.2127\end{array}$ & \\
\hline
\end{tabular}
NoTE.-The Spearman rank-order
of $\psi(L)$ and the BATSE 3B catalog. 


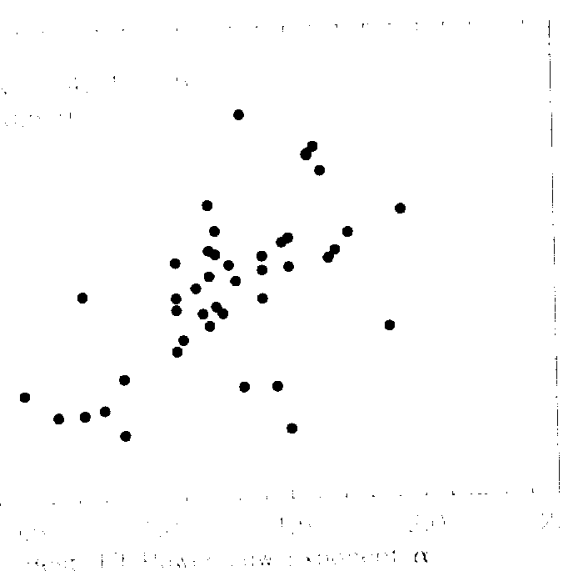

Fig. 8.-Scatter plot of the burst duration as measured by $T_{90}$ from the BATSE 3B catalog vs. the best-fit power-law exponent $\alpha$. Longer bursts tend to display larger power-law exponents and spend proportionally more time emitting at lower luminosities. These data indicate that the shorter bursts in the data set are not simply scaled-down versions of the longer bursts but instead display a different emission behavior.

Figure 8 yields a value $r_{s}=0.579$ and a corresponding $P\left(r_{s}\right.$, $N)=4.71 \times 10^{-5}$, thereby indicating that a correlation is highly probable.

The data in Figure 8 show that longer bursts (as indicated by larger values of $T_{90}$ ) tend generally to have larger best-fit power-law exponents $\alpha$; i.e., their particular $\psi(L)$ functions are steeper. Thus we observe that these longer bursts spend proportionally more time emitting energy at lower luminosities. The time profiles of short bursts are therefore not simply shortened versions of longer bursts; there is a fundamental difference in their emission behavior.

The relationship in Figure 8 between $T_{90}$ and the shape of the $\psi(L)$ distribution as characterized by the best-fit exponent $\alpha$ may be explained in a variety of ways. For example, in the context of Shaviv \& Dar (1996), at a given distance, bursts occurring in regions with a higher density of background starlight due to higher stellar volume densities may produce a brighter and longer-lived envelope of emission, i.e., a longer burst. The relative enhancement of the envelope, less intense than the peaks within the burst, would result in an overall profile that spends proportionally more time at lower luminosities.

Alternatively, Figure 8 is consistent with the hypothesis that gamma-ray bursts have an energy budget that is approximately constant for all bursts. An constant energy reservoir for all events may not be unreasonable for models involving neutron star binary systems or accretion-induced collapse of a neutron star or white dwarf (e.g., Eichler et al. 1989; Woosley 1992) because of the narrow range of masses and gravitational binding energies found in these systems. Under this assumption, bursts that spend their energy less rapidly can occur over a greater length of time, while the more spendthrift bursts deplete their energy source in a shorter time span. These are obviously not the only possible explanations for the relationship found here between the best-fit exponent to the $\psi(L)$ distribution and the duration as measured by $T_{90}$; however, they illustrate that this behavior can be explained in the context of current burst models.

\subsection{Best-Fit Exponent a versus Hardness Ratio}

We have also investigated the relationship between the best-fit exponent $\alpha$ and the hardness ratio as computed in the BATSE 3B catalog (Meegan et al. 1996). The hardness ratio is calculated in the $3 \mathrm{~B}$ catalog by taking the total burst fluence (ergs $\mathrm{cm}^{-2}$ ) in the energy range $100-300 \mathrm{keV}$ and dividing it by the fluence in the energy range $50-100 \mathrm{keV}$. Figure 9 shows a plot of the best-fit exponent $\alpha$ as a function of the 3B hardness ratio. Again, we find some statistical evidence for a correlation between these two data sets, as the Spearman test returns values of $r_{s}=0.408$ and $P\left(r_{s}\right.$, $N)=6.58 \times 10^{-3}$.

We observe in Figure 9 that bursts with large power-law exponents $\alpha$ tend to have harder overall spectra than those with smaller power-law exponents. Therefore, for the 50 brightest bursts observed by BATSE, those that spend a proportionally larger amount of time with luminosity near the peak luminosity ( $\alpha$ small) tend to possess fluence spectra with a larger proportion of low-energy photons (small hardness ratio). Conversely, those bursts spending a proportionally smaller amount of time emitting photons at a rate near the peak luminosity have fluence spectra with a generally larger proportion of high-energy photons (larger hardness ratio). With a Spearman probability slightly less than one chance in 100 , the possibility of a statistical fluctuation cannot be ignored in this case. We therefore have chosen to forgo extensive speculation over possible physical causes.

\section{3. $T_{90}$ versus $3 B$ Hardness Ratio}

In the preceding paragraphs we have shown a high probability for correlations between the best-fit exponent $\alpha$ and $T_{90}$ and to a slightly lesser extent between the best-fit exponent and the $3 \mathrm{~B}$ hardness ratio. It is therefore possible that one might find a correlation between $T_{90}$ and hardness ratio. Indeed, in the literature one can find several examples where such a correlation has been noted in the BATSE data for the ensemble of detected bursts (e.g., Kouveliotou et al. 1993). For the overall BATSE data set, one finds that the shorter bursts tend to have harder fluence spectra.

Figure 10 shows a scatter plot of $T_{90}$ versus hardness ratio for the 50 brightest bursts in the BATSE $3 \mathrm{~B}$ catalog. It is important to stress that all the data contained in Figure 10 are simply extracted from the BATSE calalog and are independent of the analysis of $\psi(L)$. A Spearman rank-order test on these data results in values of $r_{s}=0.662$ and $P\left(r_{s}\right.$, $N)=1.32 \times 10^{-6}$, significant evidence for a correlation. However, close inspection of the figure reveals that the

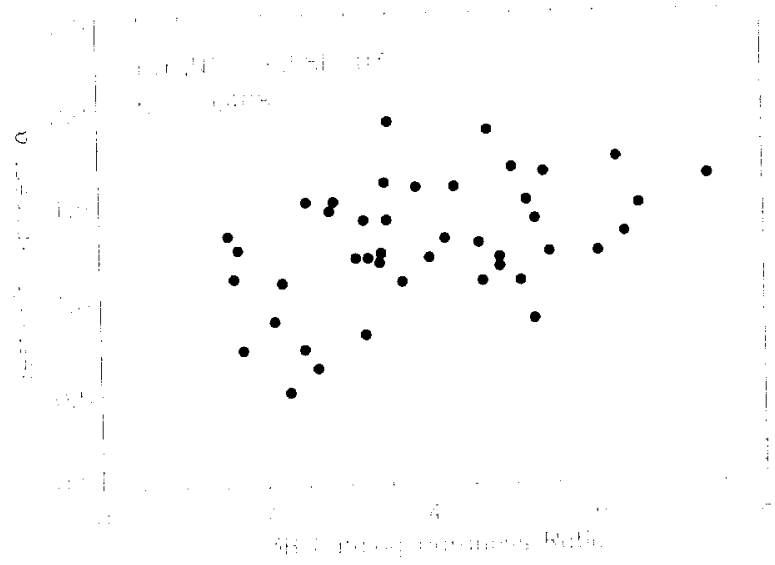

Fig. 9.-Scatter plot of the best-fit exponent $\alpha$ vs. the fluence hardness ratio from the BATSE 3B catalog. Those bursts exhibiting softer spectra have generally smaller values of $\alpha$, spending proportionally more time at higher luminosities than those bursts with hard spectra. 


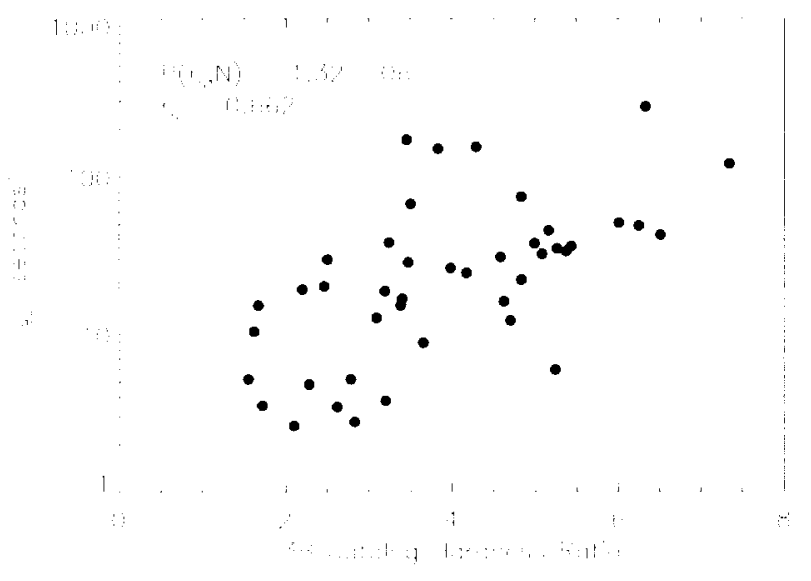

Fig. 10.-Scatter plot of the burst duration as measured by $T_{90}$ vs. the hardness ratio from the 3B catalog. These data are obtained from the burst catalog, independent of the $\psi(L)$ analyses. The low value of $P\left(r_{s}, N\right)$ indicates a high probability for correlation among these two variables. Indeed, the apparent correlation is opposite to the hardness-duration correlation for the entire burst ensemble reported elsewhere in the literature.

correlation for the 50 brightest bursts is opposite to that of the entire burst ensemble as reported by Kouveliotou et al. (1993) and others. There is no general intensity trend among the points shown in Figure 5, and Table 2 indicates that intensity is unlikely to be correlated with either $T_{90}$ or hardness ratio in this limited burst sample.

This correlation has also been independently discovered in the BATSE data and seen in gamma-ray burst data obtained by the Phebus Experiment (Dezalay et al. 1996), strong evidence that the correlation is indeed real. This behavior is also consistent with the results presented in the previous two subsections, as bursts with large values of $T_{90}$ were shown to spend a proportionally larger amount of time at low brightnesses (\$ 4.1 ), and bursts that spend proportionally larger amounts of time at low brightnesses were found to display harder fluence spectra $(\$ 4.2)$. One therefore expects to find that for the bursts employed here, those with harder fluence spectra will also show larger values of $T_{90}$, as is the case with the data shown in Figure 10.

For the analysis, we have selected bursts based on their $1.024 \mathrm{~s}$ peak fluxes. Bursts with durations significantly less than $1.024 \mathrm{~s}$ can have their fluxes underestimated by this measure of brightness, as the time interval will also include background, as well as source, emission, time. Thus by selecting bursts based on this criteria, one may introduce a bias against bursts whose duration is less than about 1 second. It is known (e.g., Fishman et al. 1994) that the distribution of $\log T_{90}$ is bimodal, with a clear separation near $T_{90} \sim 2 \mathrm{~s}$. Therefore by using the $1.024 \mathrm{~s}$ brightness measure, we have selected against bursts belonging to the leftward (short) mode of the $\log T_{90}$ distribution. These short bursts can have very hard spectra.

Figure 11 demonstrates how the inclusion of short bursts affects the correlation found in Figure 10. In this figure we plot the value of $T_{90}$ versus $3 \mathrm{~B}$ catalog hardness for the 50 brightest bursts on the $0.064 \mathrm{~s}$ timescale, where data were available. Visual comparison of the two figures shows that many bursts are common to both plots. However, in Figure 11 , the addition of bursts that are bright on $0.064 \mathrm{~s}$ peak flux scales introduces bursts with durations significantly shorter than $1 \mathrm{~s}$ and with hardness ratios that can be quite large. These bursts are found in the leftward mode of the bimodal

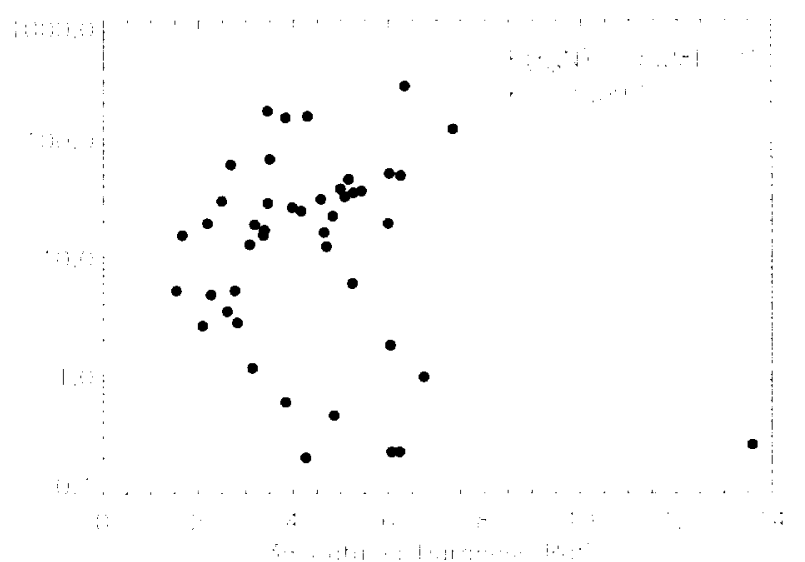

FIG. 11. Scatter plot of burst duration as measured by $T_{90}$ vs. the hardness ratio from the $3 \mathrm{~B}$ catalog for the brightest bursts measured on the $0.064 \mathrm{~s}$ peak flux timescale. The inclusion of short bursts with durations less than $\sim 1$ second and hard spectra washes out the correlation found in Fig. 10 for the same variables using bursts ranked by their $1.024 \mathrm{~s}$ peak fluxes.

$\log T_{90}$ distribution (e.g., Fishman et al. 1994). The inclusion of bursts from the lower portion of the $\log T_{90}$ distribution effectively washes out the correlation found for the bursts in Figure 10, as a Spearman test results in values of $r_{s}=0.097$ and $P\left(r_{s}, N\right)=5.28 \times 10^{-1}$.

The use of bursts ranked by intensity on shorter timescales removes the correlative effect found in Figure 10. On the one hand, this may indicate that a selection bias related to the definition of peak flux is a contributing factor to the high probability for correlation found in Figure 10. On the other hand, it may also indicate different signature from bursts in the two different modes of the $\log T_{90}$ distribution based on fundamental differences in the properties of bursts found in these two areas of the $\log T_{90}$ distribution. From this viewpoint, the behavior found in Figure 10 is an intrinsic property of the events themselves. Regardless, bright bursts with durations in excess of $\sim 2$ seconds have a relationship between their hardness ratios and durations which is opposite to that of the entire BATSE gamma-ray burst ensemble.

\section{DISCUSSION OF SYSTEMATICS}

The physical implications and/or underlying causes of these apparent correlations in the data set are not yet fully understood. We have invested some level of effort to investigate the possibility that any correlations $\left[P\left(r_{s}, N\right)\right.$ of order $10^{-3}$ or less] are not due to systematic or selection effects from the analysis or the BATSE experiment. Of the correlations found in the data set, we believe that only the low $P\left(r_{\mathrm{s}}, N\right)$ values obtained in comparing $\langle L\rangle$ to the best-fit exponent $\alpha$ and in comparing $\langle L\rangle$ to the burst intensity have nonphysical origins. It is possible that these others also have nonphysical origins; however, we have not been able to find such evidence. Within the use of the $1.024 \mathrm{~s}$ brightness scale, we believe there are no lingering intensitydependent selection biases or systematic effects, as the burst intensities appear uncorrelated with any of the computed parameters aside from the understood variation with $\langle L\rangle$ discussed above.

Although the analysis here utilizes the $1.024 \mathrm{~s}$ timescale for intensity ranking, we have also performed less detailed analyses by ranking bursts according to their 0.256 and 
$0.064 \mathrm{~s}$ peak fluxes. This was done to investigate any changes to the correlations found with the $1.024 \mathrm{~s}$ definition. As there is significant overlap between the three sets of 50 brightest bursts, the correlations found in Table 2 are present to a slightly greater or lesser quantitative degree regardless of the defining timescale for the peak flux.

The use of the $0.064 \mathrm{~s}$ DISCSC data offers the best available time resolution over the lifetime of the entire burst, thereby providing the best ability to resolve the short timescale structure that contributes to the $\psi(L)$ distribution. It should be obvious that at some point, the temporal binning of the burst profile will effect the computed $\psi(L)$, as in the extreme case where the burst is entirely contained in one time bin. The computed $\psi(L)$ in this case would be represented by a delta function located at the normalized intensity of 1.0 . We have not been able to discern a significant systematic change in the computed $\psi(L)$ or its properties using a more coarse time resolution, provided that the temporal structure of the burst profile is still well resolved on these larger timescales.

The bursts used in this analysis have very high signal-tonoise ratio in the BATSE data. Partly for this reason, we have found that changing the intervals over which the background model was computed has little to no effect on either the resulting $\psi(L)$ distribution or its relevant parameters, provided that one is reasonable in choosing both the width and location of the intervals for the quadratic fit. The use of a $3 \sigma$ lower limit has proven effective in removing noise from the distribution. In general, the presence of a noise contribution to the $\psi(L)$ distribution is easily distinguished, as it occurs at the low end of the distribution and is characterized by a slope significantly steeper than that observed over the remaining $\psi(L)$ dynamic range.

\section{CONCLUSIONS}

We have presented the first results from our study of the internal distribution of luminosity within bright gamma-ray bursts. The breadth of the $\psi(L)$ function far exceeds the range of peak luminosity observed among bright bursts (cf. Horack et al. 1994). The general shape of the luminosity distribution is found to be correlated with the burst duration as measured by $T_{90}$. The correlation between the duration and the shape of the $\psi(L)$ function is qualitatively consistent, e.g., with the recent burst model of Shaviv \& Dar (1996) or with the hypothesis that the energy reservoir is nearly constant for all bursts. However, these are merely two illustrations of how the behavior might be explained, and, indeed, other models may also offer suitable explana- tions. Regardless of the responsible mechanism, these analyses have shown that short intense bursts are not simply contracted versions of longer bright bursts. For these bright bursts, the shape of the internal luminosity distribution also appears possibly to be correlated with the fluence hardness ratio found in the BATSE 3B catalog (Meegan et al. 1996).

The existence of correlations between duration and the logarithmic slope of $\psi(L)$ and between hardness ratio and the $\psi(L)$ logarithmic slope has led to the discovery of a correlation between hardness ratio and duration. Correlations between these two parameters have been reported before (e.g., Kouveliotou et al. 1993) for the entire burst ensemble. The correlation found here is indeed strong, with a Spearman probability of $P\left(r_{s}, N\right)$ of $1.32 \times 10^{-6}$ that two uncorrelated data sets would yield an equally or more disparate $r_{s}$ value. However, for the 50 brightest bursts on the $1.024 \mathrm{~s}$ peak flux timescale in the BATSE 3B catalog, the sense of the correlation between these two parameters is opposite to that found for the entire burst ensemble. This result has also been found independently in the BATSE data by Dezalay et al. (1996) and confirmed in their analysis of data from the Phebus experiment. The correlation is not present if the selection of bright bursts is based on their $0.064 \mathrm{~s}$ peak fluxes, primarily because of the inclusion of short $\left(T_{90}<1 \mathrm{~s}\right)$ bursts that have very large hardness ratios.

A full understanding of the physical implications of these phenomenological burst properties is not yet realizable and will require significant additional in-depth study. We are proceeding with further detailed analyses. These include the extension to lower intensities, the application of selforganizing criticality analysis methods, and separation of the bursts based on the presence of emission above $300 \mathrm{keV}$, which has been found to yield one set of bursts whose intensity distribution is consistent with homogeneity and one set with a deviation from homogeneity more significant than that found in the overall population (e.g., Pendleton et al. 1996).

The authors would like to acknowledge Robert Mallozzi of the University of Alabama in Huntsville and Thomas Koshut of Universities Space Research Association for useful discussions and review of drafts of the manuscript. Jon Hakkila is supported by a Compton Gamma Ray Observatory Guest Investigator grant. The independent work of Dezalay et al. (1996) on the hardness-duration correlation of $\S 4.3$ was brought to our attention during the drafting of the manuscript.

\section{REFERENCES}

Davis, S. P., et al. 1993, in AIP Conf. Proc. 307, Gamma-Ray Bursts, ed. G. J. Fishman, K. Hurley, \& J. J. Brainerd (New York: AIP), 182

Dezalay, J.-P., et al. 1996, ÄpJ, submitted

Eichler, D., et al. 1989, Nature, 340, 126

Fishman, G. J., et al. 1989, in Proc. GRO Science Workshop, 2-89, NASA/ GSFC, Greenbelt, Maryland

Fishman, G. J., et al. 1994, ApJS, 92, 229

Fishman, G. J., \& Meegan, C. A. 1995, ARA\&A, 33, 415

Hakkila, J., et al. 1995, ApJ, 454, 134

. 1996 ApJ, 462,125

Horack. J. M. 1991. Development of the Burst and Transient Source Experiment, NASA RP-1268

Horack, J. M., et al. 1991, in AIP Conf. Proc. 265, Gamma-Ray Bursts, ed.

W. S. Paciesas \& G. J. Fishman (New York: AIP), 373

Horack, J. M., Emslie, A. G., \& Meegan, C. A. 1994, ApJ, 426, L5

Horack. J. M., et al. 1996, ApJ, 462, 131

Kouveliotou, C.. et al. 1993, ApJ, 413, L101
Link, B., Epstein, R., \& Priedhorsky, W. 1993, ApJ, 408, L81

Meegan, C. A., et al. 1992, Nature, 355, 143

Meci, 1996 , ApJ, in press

Nemiroff, R., Norris, J., Bonnell, J. J., Wickramasinghe, W. A. D. T., \& Kouveliotou, C. 1994, ApJ, 435, L133

Norris, J. P., et al. 1993a, in AIP Conf. Proc. 307, Gamma-Ray Bursts, ed. G. J. Fishman, K. Hurley, \& J. J. Brainerd (New York: AIP), 172 1993b, in AIP Conf. Proc. 307, Gamma-Ray Bursts, ed. G. J. Fishman, K. Hurley, \& J. J. Brainerd (New York: AIP), 177 Pendleton, G. N., et al. 1996, ApJ, in preparation

Press, W. H., Flannery, P. B., Teukolsky, S. A., \& Vetterling, W. T. 1989, Numerical Recipes (Cambridge: Cambridge Univ. Press)

Shaviv, N. J., \& Dar, A. 1996, astro-ph/9606032, preprint

Ulmer, A., \& Wijers, R. A. M. J. 1995, ApJ, 439, 303

Ulmer, A., Wijers, R. A. M. J., \& Fenimore, E. E. 1995, ApJ, 440, L9

Wald, A. 1939, Trans. Am. Math. Soc., 46, 280

Woosley, S. 1992, ApJ, 405, 273 